\title{
Theory of molecular crowding in Brownian hard-sphere liquids
}

\author{
Alessio Zaccone and Eugene M. Terentjev \\ Cavendish Laboratory, University of Cambridge, JJ Thomson Avenue, Cambridge CB3 OHE, United Kingdom
}

(Received 28 October 2011; published 15 June 2012)

\begin{abstract}
We derive an analytical pair potential of mean force for Brownian molecules in the liquid state. Our approach accounts for many-particle correlations of crowding particles of the liquid and for diffusive transport across the spatially modulated local density of crowders in the dense environment. Focusing on the limit of equal-size particles, we show that this diffusive transport leads to additional density- and structure-dependent terms in the interaction potential and to a much stronger attraction (by a factor of $\approx 4$ at average volume fraction of crowders $\left.\phi_{0}=0.25\right)$ than in the standard depletion interaction where the diffusive effects are neglected. As an illustration of the theory, we use it to study the size of a polymer chain in a solution of inert crowders. Even in the case of an athermal background solvent, when a classical chain should be fully swollen, we find a sharp coil-globule transition of the ideal chain collapsing at a critical value of the crowder volume fraction $\phi_{c} \approx 0.145$.
\end{abstract}

DOI: 10.1103/PhysRevE.85.061202 PACS number(s): 61.20.-p, 82.20.Uv, 34.10.+x, 95.30.Qd

\section{INTRODUCTION}

The theory of the liquid state relies heavily on the statistical mechanics of hard-sphere systems [1]. One of the most remarkable among these results is the Percus-Yevick integral equation, the solution of which yields an accurate description of the structure of hard-sphere liquids in terms of the radial distribution function $g(r)$. The latter is related to the probability of finding a second particle at a position $r$ along the outward radial coordinate measured from the test particle. $g(r)$ typically features a peak near contact (the first coordination shell) associated with an enhanced probability of finding a second particle near contact. This is due to the osmotic pressure exerted by all the other particles in the system, which is not balanced in the gap between the two particles near contact, an effect which is known as depletion attraction. This effect is clearly the result of collective dynamics ("more is different") since it introduces an attractive interaction which depends on the density and is not present originally in the pair interaction between two hard particles. In ensembles of hard spheres of equal size, this attraction induced by many-body effects is known to be weak: even at high density it is barely of the order of the thermal energy scale $k T$ [2]. This attraction, however, may become substantial in binary mixtures of hard spheres where spheres of two different sizes coexist: this is an important attractive interaction that determines the phase behavior of colloid-polymer mixtures [3], and its first theoretical description (valid for dilute systems) was given long ago in a famous work by Asakura and Oosawa [4]. This attraction plays an important role in biological systems where, e.g., it is a factor controlling cellular organization [5].

However, in both colloidal and biological systems, the particles are not simply hard spheres subject to Newtonian dynamics. Instead, they are Brownian particles obeying Langevin dynamics, and they are sensitive to the local chemical potential of the solvent [6]. Since this aspect has been neglected both in theoretical and simulation studies of depletion in the past, it is an interesting question to ask: what is the effect of the dense solvent and the diffusive nature of particle dynamics on the depletion attraction? Here we show that many-particle correlations that lead to the modulated radial distribution $g(r)$ can strongly affect the local osmotic pressure profile between two test particles. As a result, diffusive driving forces arise which push the two particles together more strongly than if the dynamics were purely Newtonian, as has been assumed in [4] and ever since. This is reflected in a much stronger depletion attraction for Brownian particles compared to hard spheres in vacuo, such that the attraction minimum can be substantial also in systems of equal-size particles.

We then apply this theory to calculate the coil-globule transition of ideal polymer chains in a solution with Brownian particles of approximately the same size as the monomers. It was observed 40 years ago that adding polyethylene glycol (PEG) molecules to solutions of DNA in water leads to the collapse or condensation of DNA as soon as a critical concentration of PEG is reached [7]. Several theories have been proposed to explain this "crowding effect," which has widespread implications as the biological function of all biopolymers is strongly dependent on their size and conformation, in particular, on whether they are in a swollen or collapsed state [8]. This stark distinction in biological function is reflected in the names of their "denatured" and "native" states of biopolymer molecules. One of the most familiar cases is the packing of DNA in a dense cellular environment and in vitro experiments where the crowding was artificially tested $[9,10]$. In proteins subtle changes of the molecular environment, instead of natural folding, could trigger their aggregation into toxic assemblies (such as the amyloid fibrils responsible for neurodegenerative diseases) [11]. It has been shown that folding can be promoted by the addition of inert crowders via a mechanism which bears many similarities to the crowding-induced globular collapse of generic polymers [12].

The effective interaction between pairs of polymer segments in the presence of quenched randomly distributed impurities is a classical problem in polymer physics, originally solved by Edwards, Muthukumar, and Baumgaertner [13,14]. When the impurities are mobile (annealed), a similar attraction also exists and is likewise due to entropic effects, this time in the form of depletion forces [15]. Despite the similarities, the mobile crowding problem has a different formulation because the effective potential in this case has to be related to the whole diffusive processes acting between the polymer segments and the Brownian crowders. The interplay between diffusion in a 
dense environment with a liquid structure and the depletion effect is the focus of our work.

The theories proposed to explain this phenomenon attempt to account for the many-particle correlations due to crowders, which induce an effective attraction between pairs of monomers in the chain [16-21]. This is reflected in a decreasing second virial coefficient upon increasing the volume fraction of crowders in the system, until the second virial coefficient finally changes sign, from positive to negative, and a transition from coil to globule of the polymer occurs [20]. None of these theories, however, account for the role of the microscopic liquid structure in this process, and they all generically attribute the increased effective attraction to the increased global (uniform) osmotic pressure exerted by the crowding particles. These approaches also neglect the role of the diffusive dynamics. In contrast, our approach uses as its input the radial distribution function of the fluid $g(r)$, which develops a modulated structure at increased densities. It thus allows us to obtain additional forces generated by diffusive transport across local gradients of the osmotic pressure (and the corresponding chemical potential). At higher densities this effect turns out to be much stronger than the standard depletion forces, and it of course vanishes in the low-density limit where one recovers the standard depletion attraction given, for equal-size spheres, by $-\ln g(r)$. The fact that all depletion-based theories underestimate the crowding effect manifests itself, for instance, in that the critical density of crowders for the polymer collapse, derived in [19], diverges to infinity when the size of the crowder particle matches the size of the polymer Kuhn length, clearly an unphysical outcome.

There are several steps in the development of our framework: (1) We first derive the analytical form of this effective pair potential between monomers by accounting for multiparticle correlations and also for the presence of the solvent, which shows an attractive well, increasing at a higher concentration of crowders. (2) This effective potential requires an expression for the radial distribution function $g(r)$, which we calculate using the currently most accurate analytical approach based on the Wertheim solution of the Percus-Yevick equations. (3) Using this effective potential, we then calculate the second virial coefficient for monomer-monomer interaction of a polymer chain in a good solvent and use a simple scheme of Flory meanfield free energy to qualitatively describe the chain collapse at a critical volume fraction of crowders. Our theory uses only one serious assumption, the local density approximation (LDA), the validity of which is carefully examined below. Therefore, this analysis can be applied to any kind of system, provided that the structure factor of the monomer-crowder mixture can be extracted from, e.g., radiation scattering experiments.

\section{DERIVATION}

Consider a Brownian particle moving in a sea of other similar Brownian particles, which all mutually interact via excluded volume (purely hard-sphere particles). We let the particles be embedded in a background solvent, which is a simple liquid providing the thermal bath. Let us take some arbitrarily chosen point of the system as the origin and let $\boldsymbol{r}$ be the distance vector of the tagged particle from the origin. In the local density approximation [1], let $\mu_{s}(\boldsymbol{r})$ and $\mu_{B}(\boldsymbol{r})$ be the chemical potentials of the solvent and of the Brownian particle, respectively. Then the local driving forces acting on a solvent molecule and on a Brownian particle are given by $\boldsymbol{F}_{s}=-\nabla \mu_{s}(\boldsymbol{r})$ and $\boldsymbol{F}_{B}=-\nabla \mu_{B}(\boldsymbol{r})$, respectively. By its definition, the spatial variation of the chemical potential is related to the variation in the free energy density per particle associated with the fact that both the Brownian particles and the background solvent molecules have a locally higher free energy in regions where the concentration of Brownian molecules and solvent molecules, respectively, is higher. As a consequence a driving force caused by the gradient of the chemical potential acts to bring Brownian molecules from the region where their concentration is higher into regions where their concentration is lower, and a similar driving force acts on the solvent molecules. At steady state, the two forces must be separately equal to zero. In fact, the two chemical potentials at constant pressure and temperature are related by the Gibbs-Duhem equation: $\rho(\boldsymbol{r}) \nabla \mu_{B}(\boldsymbol{r})+\rho_{s}(\boldsymbol{r}) \nabla \mu_{s}(\boldsymbol{r})=$ 0 , where $\rho(\boldsymbol{r})$ is the local density of Brownian particles and $\rho_{s}(\boldsymbol{r})$ is the local density of the solvent. Further, the mass conservation relation is valid: $v_{B} \rho(\boldsymbol{r})+v_{s} \rho_{s}(\boldsymbol{r})=1$, where $v_{B}$ and $v_{s}$ are the volumes of the Brownian particle and of the solvent molecule, respectively. The relevant net force which acts on the Brownian particle is the one relative to the solvent, which is obtained by subtracting the force locally experienced by the background solvent. In order to ensure that the unit volume of the background solvent is at rest with respect to the particle, we need to consider forces per unit volume instead of the bare forces [6]. Hence we have $\boldsymbol{F} / v_{B}=\boldsymbol{F}_{B} / v_{B}-\boldsymbol{F}_{s} / v_{s}$, and then the net force acting on a single particle is given by $\boldsymbol{F}=\boldsymbol{F}_{B}-\left(v_{B} / v_{s}\right) \boldsymbol{F}_{s}$. Substituting $v_{s}$ and $\rho_{s}$, after a simple algebra we obtain the following general expression for the net force acting on a Brownian particle moving in the solvent populated with other Brownian particles:

$$
\boldsymbol{F}=-\frac{1}{\rho(\boldsymbol{r})} \nabla \Pi(\rho(\boldsymbol{r}))
$$

where the local osmotic pressure is defined as $\Pi(\rho(\boldsymbol{r})) \equiv$ $-\left[\mu_{s}(\boldsymbol{r})-\mu_{s}^{0}\right] / v_{s}$, with $\mu_{s}^{0}$ being the constant chemical potential of the pure solvent that does not contain any Brownian particles. This expression for the force is a consequence of our using the LDA and is only valid when the local variation in density is smooth, that is, $|\nabla \rho / \rho| \ll 1 / \xi$, with $\xi$ being the characteristic range of oscillations of the radial distribution function $g(r)[1,22]$. It is clear (e.g., see Fig. 2) that the interesting effects of crowding-induced attraction may occur at sufficiently low densities where this LDA condition is satisfied.

Equation (1) can also be derived from dimensional arguments. In a suspension of Brownian particles, $\Pi$ is the pressure exerted by the suspension itself. Then $-\nabla \Pi$ represents the local density of force acting in the suspension [23]. If $\rho$ is the number density of particles, it follows that the force acting on average upon a single particle of the suspension is given by $-(1 / \rho) \nabla \Pi$. This force is zero whenever the system is spatially homogeneous, as it ought to be, e.g., for infinitely diluted suspensions in the ideal-gas regime. If, however, the distribution of the particles is not homogeneous, this diffusive force acts to spread out local concentration gradients. As a result of this force, the particle acquires a velocity which points along the direction of local steepest decrease of the osmotic 
pressure. The close connection with diffusive motion becomes evident upon associating it with the diffusive flux $\boldsymbol{j}=\rho \boldsymbol{u}$, where the overdamped particle velocity is $\boldsymbol{u}=(D / k T) \boldsymbol{F}$. By applying the continuity operator to this flux, $\dot{\rho}=-\nabla \cdot \boldsymbol{j}$, in the low-density limit of $\Pi=k T \rho$, one recovers the Fickian diffusion equation: $\dot{\rho}=D \nabla^{2} \rho$. Within LDA, one replaces

$$
\nabla \Pi=\nabla \rho \cdot \frac{\partial \Pi}{\partial \rho},
$$

thus obtaining the diffusion equation valid for nondilute and locally inhomogeneous systems:

$$
\frac{\partial \rho(\boldsymbol{r})}{\partial t}=D_{c}(\rho) \nabla^{2} \rho(\boldsymbol{r}) .
$$

The collective diffusion coefficient $D_{c}=(D / k T) \partial \Pi / \partial \rho$ takes care of the increased density effects [6]. This derivation highlights the fact that Eq. (1) represents the driving force acting on a Brownian particle in nondilute and locally inhomogeneous systems and is consistent with the particle migration being governed by diffusion, which in turn is driven by gradients of the chemical potential.

Let us now consider that the center of frame from which $\mathbf{r}$ is measured coincides with a test particle and consider a second particle approaching the test particle in the moving spherical coordinates frame centered on the test particle. The work required to bring the second particle to a point $\boldsymbol{r}$ measured in the moving frame of the first particle is given by

$$
W=-\int \boldsymbol{F} \cdot d \boldsymbol{r}=\int_{\infty}^{r} \frac{1}{\rho(r)} \frac{d}{d r} \Pi(\rho(r)) d r .
$$

In the second equality we used Eq. (1) and the fact that with hard spheres the distribution of particles is spatially isotropic; thus only the radial distance $r=|\boldsymbol{r}|$ between the two monomers is a variable of the effective pair-potential energy $V_{\text {eff }}(r) \equiv W$ acting between the two Brownian monomers. To evaluate $V_{\text {eff }}(r)$ we need an equation of state for the crowders to relate the osmotic pressure and the density, and we will assume that this relation also applies locally, which, once more, amounts to making use of the LDA. For the equation of state we can use the Carnahan-Starling (CS) equation of state, which is accurate up to quite high liquid densities [1], and we write it as

$$
\frac{1}{k T} \frac{\Pi(\rho(r))}{\rho(r)}=\frac{1+\phi(r)+\phi(r)^{2}-\phi(r)^{3}}{[1-\phi(r)]^{3}},
$$

where $\phi(r)=\left(\pi \sigma^{3} / 6\right) \cdot \rho(r)$ is the volume (packing) fraction of crowders ( $\sigma$ is chosen as the diameter of the Brownian particle). The CS equation of state is frequently used in standard approaches for the calculation of depletion forces. However, we should remark that in those cases the aim is to account for density effects on the free energy of interaction, which for Newtonian hard spheres is a purely mechanical effect. That is, one takes the work $W \propto \Pi \cdot V_{d}$, where $V_{d}$ is the depletion volume (a function of $\boldsymbol{r}$ ), and the CS equation is used to relate the global ( $\boldsymbol{r}$-independent) osmotic pressure of the solution $\Pi$ to the overall density of the crowders $\phi_{0}$. In those approaches $\Pi$ and $\phi$ are not allowed to vary with $\boldsymbol{r}$ and therefore the driving force in Eq. (1) due to local variation in particle density is neglected. See Fig. 1 for a visual explanation of this contrast.

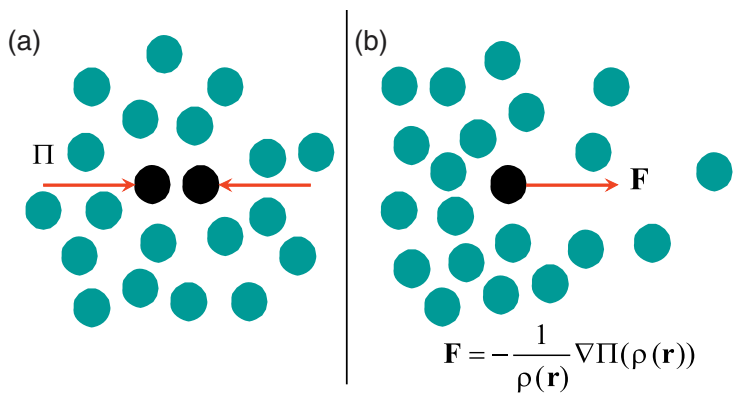

FIG. 1. (Color online) (A) The classical depletion effect when no third particle can be accommodated in the gap between the two tagged particles. As a consequence, the particles are pushed towards each other by a force proportional to the global osmotic pressure of the suspension $\Pi$, which remains unbalanced in the gap. (B) The generic effect of the diffusive driving force, Eq. (1), proportional to the local gradient of the osmotic pressure and driving the particle down the concentration gradient. This force must also be active on the two test particles of (A) because upon moving closer together they are effectively moving across a local concentration gradient of a dense fluid of crowders.

Using this CS expression (5) in the right-hand side of Eq. (4) and integrating, we obtain

$$
\frac{V_{\mathrm{eff}}(r)}{k T}=-\frac{\phi(r)-3}{[\phi(r)-1]^{3}}+\frac{\phi(r)-3}{\left(\phi_{0}-1\right)^{3}}-\ln \frac{\phi(r)}{\phi_{0}},
$$

where it is important to distinguish the local packing fraction of crowders $\phi(r)$ from the mean fraction $\phi_{0}$ at $r \rightarrow \infty$. By construction, this is an effective pair potential of interaction between two Brownian particles. In addition to the unbalanced osmotic pressure in the depletion gap near contact (which is expressed by the standard $-\ln \left[\phi(r) / \phi_{0}\right] \equiv-\ln g(r)$ term), it accounts also for the diffusive drift pushing the two particles against each other because of the local liquid structure, expressed by the two fractions in Eq. (6).

Equation (6) is the key result of this work. Note that the $-\ln g(r)$ term represents the depletion effect, which many of the earlier important publications have investigated thoroughly $[4,12,18]$. For dilute systems we have that the radial density profile around the monomer tends to become flat, $\phi(r) \rightarrow \phi_{0}$ for any $r$. In this limit, one can readily check via l'Hôpital's rule that $\lim _{\phi \rightarrow 0} V_{\text {eff }}=-k T \ln g(r)$. This limit is a well-known law of statistical mechanics [1] which holds in the dilute ideal-gas limit independently of the particular form of the pair potential. From another perspective, since this theory describes the effective pair potential between particles of a "Brownian fluid," this result means that in the dilute limit it does not matter whether the particles are Brownian or not, which is an important observation on its own. Further, our result extends the fundamental potential of mean force from the standard simple fluids to the Brownian fluids.

As we have explained above, depletion alone underestimates the effective attraction in Brownian systems, which is explicitly illustrated by plotting the two forms of effective potential in Fig. 2, after substituting the explicit form of $g(r)$ at a given mean density $\phi_{0}$, as explained in the Appendix [24]. This effective pair potential is plotted for $\phi_{0}=0.25$, which is a relatively high density still well within the fluid region of the phase diagram of hard spheres. It is evident that the effective 


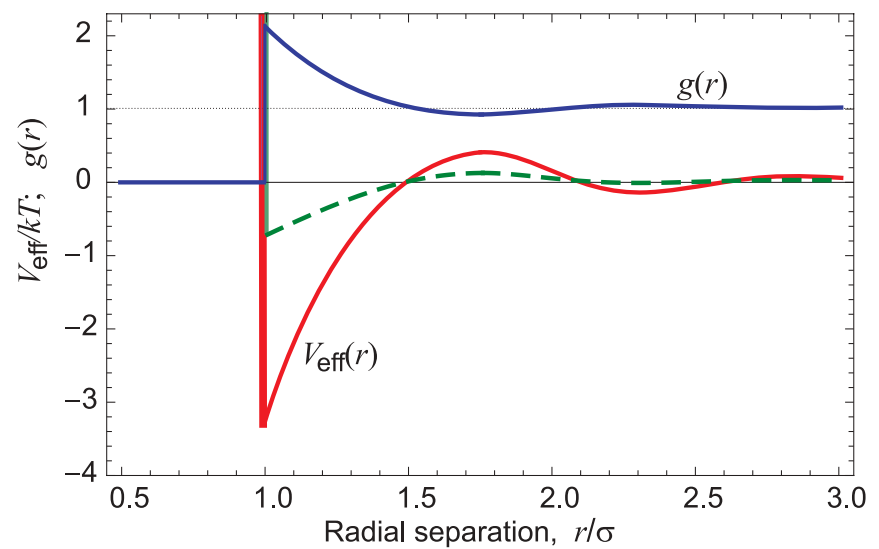

FIG. 2. (Color online) Radial distribution function and effective pair potential for $\phi_{0}=0.25$ calculated using the Percus-Yevick theory [24]. There are no free parameters in this athermal system with only excluded volume between particles in contact. The dashed line shows the prediction of the depletion theory, $-\ln g(r)$, for comparison.

potential develops a short-range attractive well aligned with the first coordination shell of the radial distribution function. It is also clear that the attraction minimum is significantly deeper, by a factor $\simeq 4$, than the one given by the standard depletion potential $-\ln g(r)$ alone, which suffices to describe hard spheres in vacuo (that is, ignoring the diffusion of particles in the suspending matrix). The two new terms in the right-hand side of Eq. (6) turn out to be a major effect not captured by earlier theories.

Figure 3 plots the evolution of the effective potential $V_{\text {eff }}(r)$ on increasing the mean concentration of crowders in a solution, illustrating that the depth of the attractive well exceeds $k T$ at $\phi_{0} \geqslant 0.15$. It is interesting to note that also the local maximum, i.e. the energy barrier corresponding to the local minimum in the $g(r)$, is significantly larger for Brownian particles than for Newtonian hard spheres. This can be understood by recalling that the diffusive driving force acts to push the particles down the $\rho$ and $\Pi$ gradients. Upon approaching each other the two particles are separated by a (spherical) "monolayer" of third particles of the first coordination shell. In order to come closer together, the two particles need to diffuse "uphill," i.e.

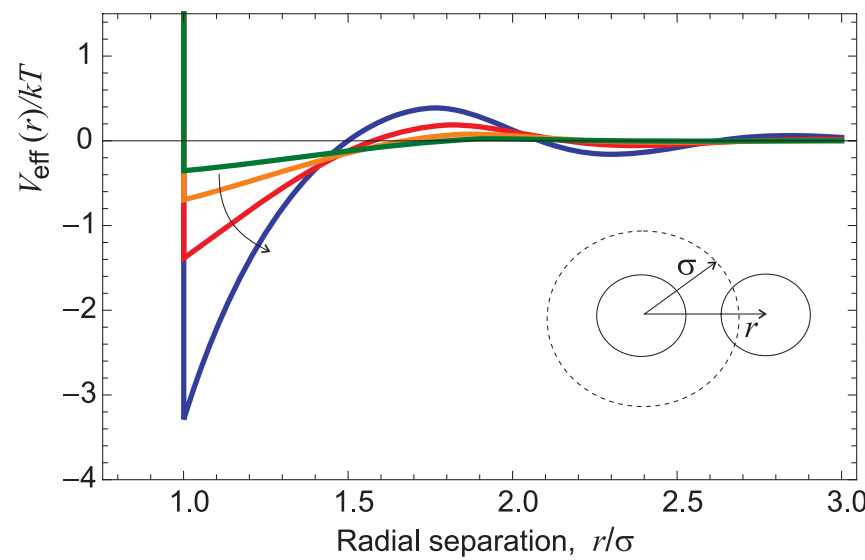

FIG. 3. (Color online) The effective potential upon increasing the mean volume fraction of Brownian crowders; $\phi_{0}=0.1,0.15,0.2$, and 0.25 for the successive curves.

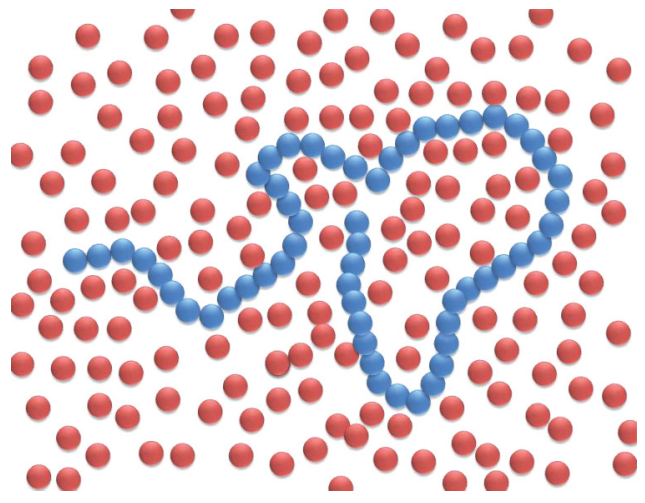

FIG. 4. (Color online) Schematic representation of the model used: an excluded-volume chain in a suspension of Brownian particles. The background solvent, taken to be structureless, is not shown.

jump over the particle in between. In this case the diffusive driving force is pointing outwards along $r$, and it generates an additional repulsion with respect to the bare local osmotic pressure, which is reflected in the higher local maximum of $V_{\text {eff }}$ compared to the case of hard spheres in vacuo. This effect is particularly important for diffusion-limited reaction kinetics in crowded systems [25-27]).

\section{COIL-GLOBULE TRANSITION DRIVEN BY CROWDING}

Let us now imagine that the two interacting particles represent two monomers of a polymer chain in a suspension of other Brownian molecules (crowders) which interact with the two test monomers via excluded volume. The model is sketched in Fig. 4. For this system, we note that the effective interaction derived above is a function of the overall density in the system, which includes both the monomers and the crowders. In a more precise way we could decompose the two contributions such that

$$
g(r) \equiv \frac{\phi(r)}{\phi_{0}}=\frac{\phi_{m c}(r)}{\phi_{\mathrm{tot}}}+\frac{\phi_{m m}(r)}{\phi_{\mathrm{tot}}},
$$

where $\phi_{m c}$ is the local profile of crowders around the test monomer, $\phi_{m m}$ is that of other monomers, and $\phi_{\text {tot }}$ is the overall bulk packing fraction. At a high density of crowders, $\phi_{m c} \gg$ $\phi_{m m}$ and the general radial distribution function is determined by crowders only, $g(r) \approx \phi_{m c}(r) / \phi_{0}$, while in the opposite limit of $\phi_{m c} \rightarrow 0$ we have $g(r) \approx \phi_{m m}(r) / \phi_{0}$, that is, only determined by the hard-sphere interaction of monomers. We can write down the effective second virial coefficient $B_{2}$ for the two chosen polymer segments. Normally, one expects $B_{2}$ to be a function of $T$ (as well as the mean concentration $\phi_{0}$ ); however, in our case this expression is purely entropic and therefore completely athermal:

$$
B_{2}=2 \pi \int_{0}^{\infty} r^{2}\left[1-e^{-V_{\text {eff }}(r) / k T}\right] d r .
$$

The sign of the second virial coefficient controls whether the polymer chain would be found in the collapsed state, which corresponds to $B_{2}<0$, or would be swollen $\left(B_{2}>0\right)$ [28], as we are going to discuss below. For a given $\phi_{0}$ the sign 


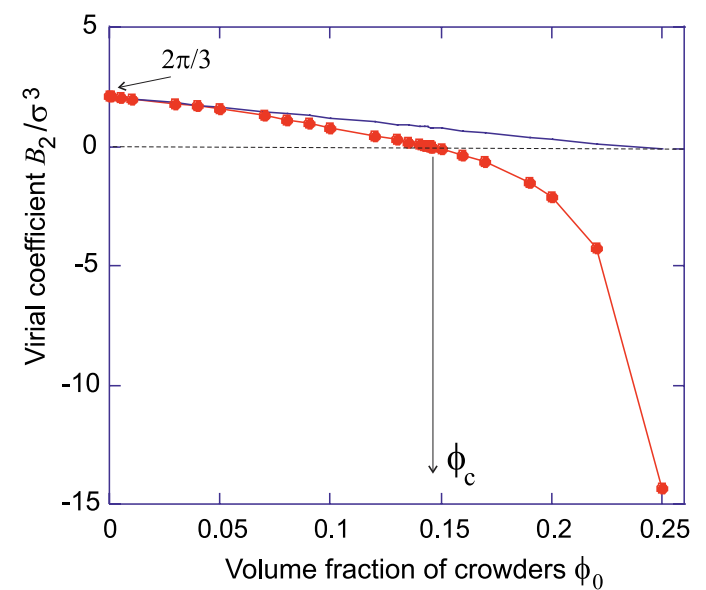

FIG. 5. (Color online) The values of $B_{2}$ calculated numerically from Eq. (8), showing the point of changing sign at a critical concentration $\phi_{c} \approx 0.145$. The straight line illustrates the linearorder approximation, $B_{2} \approx\left(2 \pi \sigma^{3} / 3\right)\left[1-(17 / 4) \phi_{0}\right]$, which strongly overestimates the critical point $\phi_{c}$.

of $B_{2}$ is dictated by the balance in the integrand between the repulsive hard-core part of the effective potential (for spheres of diameter $\sigma, B_{2}=2 \pi \sigma^{3} / 3$ ) and the attractive part due to the unbalanced osmotic pressure. As the hard-core part is independent of $\phi_{0}$, the behavior of $B_{2}$ as a function of the volume fraction of crowders is therefore controlled by the behavior of the attractive part of $V_{\text {eff }}(r)$ as a function of $\phi_{0}$. As shown in Fig. 3, the strength of the attractive part of $V_{\text {eff }}(r)$ is a monotonically increasing function of $\phi_{0}$. This is intuitively expected because the unbalanced osmotic pressure in the excluded volume gap between two monomers (the standard depletion) and the diffusive driving force both become stronger with increasing density of crowders. Figure 5 shows the evolution of $B_{2}$ with an increasing concentration of crowders, together with the linear-order approximation valid at very low densities, which is easily obtained by the series expansion of Eqs. (6) and (8). There is a remarkable similarity with the traditional $T$ dependence of $B_{2}(T)$ for the Lennard-Jones type of attractive-repulsive potentials; however, we need to emphasize once again that our basic theory is purely entropic and therefore athermal. The application of the effective potential $V_{\text {eff }}$ from Eq. (6) to evaluate $B_{2}$ leads to a change of the sign of the second virial coefficient in a system of purely hard-core repulsive particles when a critical concentration $\phi_{c} \approx 0.145$ is reached.

As a simple exercise to illustrate the theory we take the Flory excluded-volume theory of polymer chains, where the free energy is given by the two contributions determined by the size (radius of gyration $R$ ) of the chain [28], the total potential energy of effective pair interactions and the entropy of configurations of an ideal (Gaussian) random walk:

$$
F \simeq k T\left(B_{2} \frac{N^{2}}{R^{3}}+\frac{R^{2}}{N b^{2}}\right),
$$

where $N$ is the number of monomers in the chain and $b$ is the linear size of the monomer. To simplify the key qualitative results, let us assume $b=\sigma$, that is, the size of the chain segments and the crowders is the same, as illustrated in

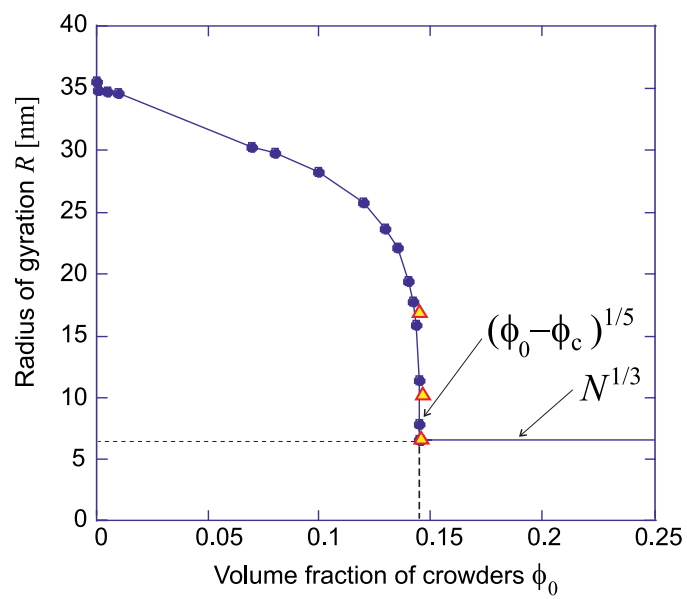

FIG. 6. (Color online) The size of the polymer as a function of the volume fraction of molecular crowders for $b=\sigma=1 \mathrm{~nm}$ and number of monomers $N=300$. The critical transition occurs at the point $\phi_{0}=\phi_{c}$, where the second virial coefficient $B_{2}$ changes its sign. At a high concentration the collapsed polymer chain is assigned a dense-packed size $R \simeq b N^{1 / 3}$. The additional data points (triangles) have been calculated using the perturbation expansion for excluded volume.

Fig. 4. In spite of its many conceptual shortcomings, the Flory expression (9) is remarkably accurate. Although it neglects correlations between monomers along the chain, the errors in the internal energy and the entropic terms are roughly the same, which leads to the cancellation of errors, which is responsible for the good accuracy of this crude model [28]. Minimizing Eq. (9) with respect to $R$ leads to the classical estimate of the size of the polymer chain swollen in a good solvent:

$$
R \simeq\left(B_{2} b^{2} N^{3}\right)^{1 / 5}
$$

which applies for $B_{2} \geqslant 0$. When $B_{2}<0$ the free energy (9) does not have a well-defined minimum, and the chain is considered collapsed into a dense globule. The theory of polymer collapse in a poor solvent is developed [29-31], and it is known that to properly describe the globular (collapsed) state one needs to invoke the third virial coefficient contribution to the interaction energy, which provides the stabilizing effect against an apparent collapse to a point, $R \rightarrow 0$. In this paper we do not deal with the globular state and the considerable computational difficulties associated with it. Our aim is merely to illustrate the effect of molecular crowding on a chain in an otherwise good solvent and to describe the onset of the chain collapse, which occurs at $B_{2} \geqslant 0$ where $B_{3}$ is not yet relevant.

Using Eq. (6) for the effective potential evaluated making use of the Percus-Yevick theory for $g(r)$ [24] in Eq. (8), and Eq. (8) in Eq. (9), the polymer size as a function of the crowder concentration $\phi_{0}$ has been evaluated for $b=\sigma$ and is plotted in Fig. 6. The power law scaling with the crowder density follows directly from Eq. (10) when one substitutes the linear relation $B_{2} \approx$ const $\times\left(\phi_{c}-\phi_{0}\right)$ near the transition point.

One may argue that the characteristic scaling of the critical point might change with the third virial coefficient taken into account. Therefore, we also estimated the polymer size based on the perturbative expansion by Muthukumar and Nickel [32], which accounts for the effect of the three-body collisions and 
for chain connectivity in a simple way. The perturbation is done on the distribution function of the end-to-end vector using $B_{2}$ as the small parameter near the point of its changing sign. The full expression is reported as Eq. (2.100) in [33], where, strictly, the perturbation expansion parameter is $z=(3 / 2 \pi)^{3 / 2} B_{2} N^{1 / 2} / b^{3}$. A few points calculated at $\left(\phi_{0}-\phi_{c}\right) \rightarrow 0$ with this approach are shown in Fig. 6 and are found to effectively coincide with the more naive Flory calculation. Hence we will stick to the handier Flory description to analyze the physics predicted by the model.

The theory predicts a sharp collapse of the polymer at a well-defined critical value of the crowder volume fraction, at $\phi_{c} \simeq 0.145$, which is the point at which $B_{2}$ changes sign and becomes negative. This value is close to the result of [34], where it was experimentally found that DNA, under conditions of screened electrostatics, collapses sharply when $10 \%-20 \%$ of PEG is added to the solution. In the limit of an infinite dilution of crowders, $\phi_{0}=0$, the effective potential for the monomers reduces to the pure hard-core interaction and $B_{2}=2 \pi b^{3} / 3$ (since we treat the background solvent as a structureless continuum), and thus we recover the limit of a fully swollen self-avoiding chain in athermal solvents: $R=(2 \pi / 3) b N^{3 / 5}$. Previous approaches treat the crowders like Newtonian hard spheres with a uniform local density and therefore underestimate the attraction. In this context, the theory of [20] predicts no collapse for systems where crowders and monomers are comparable in size, contrary to the evidence of collapse also in that limit from experiments [34].

In the present study we have focused, to preserve the analytical clarity of the key points, on crowding molecules which have a size comparable to the size of the monomers in the polymer chain. In future studies, this theory should be extended to arbitrary monomer-crowder size ratios. The theory in fact accounts for this ratio through the radial distribution function $g(r)$ of crowders around a monomer, which can be extended to the case of arbitrary size ratios by means of a theory by Lebowitz [35]. Unfortunately, this can be done only numerically. However, we can anticipate that, upon increasing the size of the crowders, the packing around the monomer becomes less efficient and the first peak in the $g(r)$ gets smaller at the same mean packing fraction $\phi_{0}[1,35]$. This means that the collapse transition occurs at higher $\phi_{0}$ upon increasing the crowder size with respect to the monomer. The reverse occurs upon decreasing the crowder-monomer size ratio: the first peak is higher at comparatively lower $\phi_{0}$, and therefore the collapse occurs at lower $\phi_{0}$ too. Another interesting direction would be to extend this theory to specific practically relevant biomolecular systems by simply adding the specific interactions (hydrophobic, hydrogen-bonding, etc., as well as the chain bending rigidity) that are relevant in each case, e.g., in packing the DNA or (mis)folding proteins. These additional potentials act as perturbations by shifting the critical concentration of the coil-globule collapse, but the effect of crowding is still controlled by the effective two-particle potential $V_{\text {eff }}(r)$, which is essentially entropic and excluded-volume driven. Previous approaches [20] omit the microscopic details of the local structure induced by the crowders and therefore are not suitable for a realistic description of the interaction chemistry beyond the hard-sphere interaction.

\section{CONCLUSIONS}

In summary, we have developed a theory that analytically describes the effective interaction between two particles in Brownian hard-sphere liquids. In addition to the multiparticle correlations which induce the depletion attraction in hard spheres in vacuo (governed by Newtonian dynamics), we also account for the diffusive driving force acting on the two Brownian particles due to the local gradients of osmotic pressure and chemical potential dictated by the liquid-like structure. In fact, when two particles move into the depletion gap (near-contact region), they are subject not only to the unbalanced osmotic pressure in the gap (the cause of the standard depletion attraction) but also to the additional diffusive driving force which pushes them towards low-density regions (the depletion gap), thus enhancing the depletion attraction. Slightly farther apart, the particles also experience a much higher repulsive barrier where the local density decreases between the first and the second coordination spheres. The effective potential $V_{\text {eff }}(r)$ uses the radial distribution function $g(r)$ to calculate the forces between the pair of particles mediated by the structured dense fluid dominated by Brownian motion.

It turns out that this additional effect can be very substantial in systems of equal-size particles, where it increases the attraction by over a factor of 4 (at $\phi_{0}=0.25$ ) in comparison to the standard depletion interaction term $-\ln g(r)$ for hard spheres in vacuo. The latter form is correctly recovered in the low density limit $\phi_{0} \rightarrow 0$, where the local gradients of density and osmotic pressure disappear and there is no difference between hard spheres in vacuo and Brownian hard spheres.

As an illustration of the theory, we applied it to calculate the size of a polymer chain in a solution with Brownian particles (crowders) of a size equal or comparable to the monomers. Our theory predicts a sharp coil-globule transition occurring at a packing fraction of crowders $\phi_{c} \approx 0.145$, which is the volume fraction at which the second virial coefficient of the monomers changes sign owing to the effective pair potential. This approach is fully microscopic because it uses as input the radial distribution function of the monomer-crowder system, which is potentially capable of accounting for nontrivial chemistry details, whereas earlier approaches to depletion cannot go beyond the hard-sphere interaction between monomers and crowders. The enhanced attraction due to the diffusive drift in a structured environment of crowders leads to the collapse of the polymer, in agreement with simulation and experimental results, whereas previous studies neglecting the diffusive effect (and thus underestimating the attraction) predict that collapse cannot occur in that limit.

\section{ACKNOWLEDGMENTS}

This work has been supported by the EPSRC TCM Programme grant and by an Ernest Oppenheimer Fellowship.

\section{APPENDIX: ANALYTICAL FORM OF $g(r)$}

In order to calculate the potential of mean force between monomers, we need to evaluate the radial distribution function $g(r)$. It is possible to do so analytically for the case of spherical crowders which have a size comparable to the monomers and interact purely by excluded volume. In this case $g(r)$ is the one 
for hard-sphere fluids at given density $\rho \sigma^{3}$ and volume fraction $\phi_{0}=\pi \rho \sigma^{3} / 6$. The following analytical representation of $g(r)$ has been used for our calculations, which is based on the analytical solution of the Percus-Yevick equations by Wertheim [36] and the successive improved parametrizations by Trokhymchuk, Nezbeda, Jirsak, and Henderson [24]. According to the work of [24], $g(r)$ can be constructed by splitting it into a depletion part valid for $\sigma<r<r^{*}$ and a structural part valid for $r>r^{*}$. The depletion part describes the first coordination shell up to the first (depletion) minimum, whereas the structural part describes the damped oscillations at a farther distance. The analytical expressions read as follows [24,37]:

$$
\begin{aligned}
g(r)= & \frac{A}{r} e^{\mu(r-\sigma)}+\frac{B}{r} \cos [\beta(r-\sigma)+\gamma] e^{\alpha(r-\sigma)} \\
& \text { for } \sigma<r<r^{*} \\
= & 1+\frac{C}{r} \cos [\omega r+\delta] e^{-\kappa r} \quad \text { for } r>r^{*}
\end{aligned}
$$

The parameters which figure in the above expressions are all ultimately functions of $\phi_{0}$ only and can be evaluated using the improved parameterizations of [24] based on thermodynamic consistency arguments. The parameters read as

$$
\begin{gathered}
A=\sigma g_{\sigma}-B \cos \gamma, \\
B=\frac{g_{m}-\left(\sigma g_{\sigma} / r^{*}\right) e^{\mu\left(r^{*}-\sigma\right)}}{\cos \left[\beta\left(r^{*}-\sigma\right)+\gamma\right] e^{\alpha\left(r^{*}-\sigma\right)}-\cos [\gamma] e^{\mu\left(r^{*}-\sigma\right)}} r^{*}, \\
C=\frac{r^{*}\left(g_{m}-1\right) e^{\kappa r^{*}}}{\cos \left[\omega r^{*}+\delta\right]}, \\
\delta=-\omega r^{*}-\arctan \left[\left(\kappa r^{*}+1\right) /\left(\omega r^{*}\right)\right],
\end{gathered}
$$

where

$$
\begin{gathered}
g_{\sigma}=\frac{1}{4 \phi_{0}}\left(\frac{1+\phi_{0}+\phi_{0}^{2}-2 / 3 \phi_{0}^{3}-2 / 3 \phi_{0}^{4}}{\left(1-\phi_{0}\right)^{3}}-1\right) \\
g_{m}=1.0286-0.6095 \phi_{0}+3.5781 \phi_{0}^{2}-21.3651 \phi_{0}^{3} \\
+42.6344 \phi_{0}^{4}-33.8485 \phi_{0}^{5} \\
r^{*} \sigma=2.0116-1.0647 \phi_{0}+0.0538 \phi_{0}^{2} \\
\alpha \sigma=44.554+79.868 \phi_{0}+116.432 \phi_{0}^{2}-44.652 e^{2 \phi_{0}}, \\
\beta \sigma=-5.022+5.857 \phi_{0}+5.089 e^{-4 \phi_{0}}
\end{gathered}
$$

$$
\gamma=\arctan \left[-\frac{1}{\beta_{0}} \frac{\sigma\left[\alpha_{0}\left(\alpha_{0}^{2}+\beta_{0}^{2}\right)-\mu\left(\alpha_{0}^{2}-\beta_{0}^{2}\right)\right]\left(1+\frac{1}{2} \phi_{0}\right)+\left(\alpha_{0}^{2}+\beta_{0}^{2}-\mu \alpha_{0}\right)\left(1+2 \phi_{0}\right)}{\sigma\left(\alpha_{0}^{2}+\beta_{0}^{2}-\mu \alpha_{0}\right)\left(1+\frac{1}{2} \phi_{0}\right)-\mu\left(1+2 \phi_{0}\right)}\right],
$$

$$
\begin{aligned}
\alpha_{0} \sigma & =\frac{2 \phi_{0}}{1-\phi_{0}}\left(-1+\frac{d}{4 \phi_{0}}-\frac{\phi_{0}}{2 d}\right), \\
\beta_{0} \sigma & =\frac{2 \phi_{0}}{1-\phi_{0}} \sqrt{3}\left(-\frac{d}{4 \phi_{0}}-\frac{\phi_{0}}{2 d}\right), \\
\mu \sigma & =\frac{2 \phi_{0}}{1-\phi_{0}}\left(-1-\frac{d}{2 \phi_{0}}+\frac{\phi_{0}}{d}\right),
\end{aligned}
$$

$d=\left[2 \phi_{0}\left(\phi_{0}^{2}-3 \phi_{0}-3+\sqrt{3\left(\phi_{0}^{4}-2 \phi_{0}^{3}+\phi_{0}^{2}+6 \phi_{0}+3\right)}\right)\right]^{1 / 3}$.

Furthermore, the parameters $\kappa$ and $\omega$ have been evaluated according to the parametrization of Roth et al. [38] and read as follows:

$$
\begin{gathered}
\kappa \sigma=4.674 e^{-3.935 \phi_{0}}+3.536 e^{-56.270 \phi_{0}}, \\
\omega \sigma=-0.682 e^{-24.696 \phi_{0}}+4.720+4.450 \phi_{0} .
\end{gathered}
$$

[1] J. P. Hansen and I. R. McDonald, Theory of Simple Liquids (Academic, London, 2006).

[2] M. Dijkstra, R. van Roij, and R. Evans, Phys. Rev. E 59, 5744 (1999).

[3] T. Biben, P. Bladon, and D. Frenkel, J. Phys. Condens. Matter 8, 10799 (1996); R. Dickman, P. Attard, and V. Simonian, J. Chem. Phys. 107, 205 (1997); A. Zaccone, H. Wu, and E. Del Gado, Phys. Rev. Lett. 103, 208301 (2009).

[4] S. Asakura and F. Oosawa, J. Chem. Phys. 22, 1255 (1954); R. Roth, R. Evans, and S. Dietrich, Phys. Rev. E 62, 5360 (2000).

[5] D. Marenduzzo, K. Finan, and P. R. Cook, J. Cell. Biol. 175, 681 (2006).

[6] J. Dhont, An Introduction to Dynamics of Colloids (Elsevier, Amsterdam, 1996).
[7] L. Lerman, Proc. Natl. Acad. Sci. USA 68, 1886 (1971).

[8] V. A. Bloomfield, Curr. Opin. Struct. Biol. 6, 334 (1996); Biopolymers 44, 269 (1997).

[9] V. B. Teif and K. Bohinc, Prog. Biophys. Mol. Biol. 105, 208 (2011).

[10] L. Tortora and O. D. Lavrentovich, Proc. Natl. Acad. Sci. USA 108, 5163 (2011).

[11] T. P. J. Knowles, C. A. Waudby, G. L. Devlin, S. I. A. Cohen, A. Aguzzi, M. Vendruscolo, E. M. Terentjev, M. E. Welland, and C. M. Dobson, Science 326, 1533 (2009).

[12] M. S. Cheung, D. Klimov, and D. Thirumalai, Proc. Natl. Acad. Sci. USA 102, 4753 (2005).

[13] S. Edwards and M. Muthukumar, J. Chem. Phys. 89, 2435 (1988). 
[14] A. Baumgartner and M. Muthukumar, J. Chem. Phys. 87, 3082 (1987).

[15] M. R. Shaw and D. Thirumalai, Phys. Rev. A 44, R4797 (1991).

[16] A. Vrij, Pure Appl. Chem. 48, 471 (1976).

[17] A. Yu. Grosberg, I. Ya. Erukhimovitch, and E. I. Shakhnovitch, Biopolymers 21, 2413 (1982).

[18] R. P. Sear, Phys. Rev. E 58, 724 (1998).

[19] P. van der Schoot, Macromolecules 31, 4635 (1998).

[20] M. Castelnovo and W. M. Gelbart, Macromolecules 37, 3510 (2004).

[21] J. S. Kim and I. Szleifer, J. Phys. Chem. C 114, 20864 (2010).

[22] J. L. Barrat and J. P. Hansen, Basic Concepts for Simple and Complex Liquids (Cambridge University Press, Cambridge, 2003).

[23] Ya. B. Zeldovich, A. A. Ruzmaikin, and D. D. Sokoloff, The Almighty Chance (World Scientific, Singapore, 1990).

[24] A. Trokhymchuk et al., J. Chem. Phys. 123, 024501 (2005).

[25] N. Dorsaz, C. De Michele, F. Piazza, P. De Los Rios, and G. Foffi, Phys. Rev. Lett. 105, 120601 (2010).

[26] J. Dzubiella, and J. A. McCammon, J. Chem. Phys. 122, 184902 (2005).
[27] A. Zaccone, N. Dorsaz, F. Piazza, C. De Michele, M. Morbidelli, and G. Foffi, J. Phys. Chem. B 115, 7383 (2011).

[28] M. Rubinstein and R. H. Colby, Polymer Physics (Oxford University Press, Oxford, 2003).

[29] T. A. Vilgis, Phys. Rep. 336, 167 (2000).

[30] A. L. Kholodenko and K. F. Freed, J. Phys. A 17, 2703 (1984).

[31] V. G. Rostiashvili, G. Migliorini, and T. A. Vilgis, Phys. Rev. E 64, 051112 (2001).

[32] M. Muthukumar and B. G. Nickel, J. Chem. Phys. 80, 5839 (1984).

[33] M. Doi and S. F. Edwards The Theory of Polymer Dynamics (Clarendon, Oxford, 1986).

[34] V. V. Vasilevskaya, A. R. Khokhlov, Y. Matsuzawa, and K. Yoshikawa, J. Chem. Phys. 102, 6595 (1995).

[35] J. L. Lebowitz, Phys. Rev. 133, 895 (1964).

[36] M. S. Wertheim, Phys. Rev. Lett. 10, 321 (1963).

[37] A. Trokhymchuk, I. Nezbeda, J. Jirsák, and D. Henderson, J. Chem. Phys. 124, 149902 (2006).

[38] R. Roth, R. Evans, and S. Dietrich, Phys. Rev. E 62, 5360 (2000). 